\title{
KIT mutations in ocular melanoma: frequency and anatomic distribution
}

\author{
Michelle L Wallander ${ }^{1}$, Lester J Layfield ${ }^{2}$, Lyska L Emerson², Nick Mamalis ${ }^{3}$, \\ Don Davis ${ }^{3}$, Sheryl R Tripp ${ }^{1}$ and Joseph A Holden ${ }^{2}$ \\ ${ }^{1}$ ARUP Institute for Clinical and Experimental Pathology, ARUP Laboratories, Salt Lake City, UT, USA; \\ ${ }^{2}$ Department of Pathology, University of Utah School of Medicine, Salt Lake City, UT, USA and ${ }^{3}$ Department \\ of Ophthalmology and Visual Sciences, University of Utah School of Medicine, Salt Lake City, UT, USA
}

\begin{abstract}
KIT mutations are known to occur in $\sim 15 \%$ of chronic sun damaged cutaneous, mucosal, and acral melanomas. Melanomas with demonstrated activating mutations in $K I T$ or platelet-derived growth factor receptor $A$ (PDGFRA) may benefit from treatment with tyrosine kinase inhibitors. Currently, the limited data regarding KIT mutational status in ocular melanoma suggest that activating mutations are extremely rare. PDGFRA mutational status in ocular melanoma has not been determined. Seventy-five ocular melanomas (53 choroidal, 6 iris, 11 ciliary body, and 5 conjuctival) were selected from the files of the Department of Ophthalmology. High-resolution melting curve analysis and sequencing were performed to detect mutations in KIT exons 9,11 , 13, and 17 and PDGFRA exons 12 and 18. Results of mutational analysis were correlated with anatomical site and $K I T$ (CD117) immunohistochemistry. Eight of $75(11 \%)$ ocular melanomas contained mutations in either the KIT or PDGFRA gene. Five of $53(9 \%)$ choroidal melanomas were associated with mutations $(K I T$ exon $11=3$; $K I T$ exon $17=1$; PDGFRA intron $18=1)$. Two of six $(33 \%)$ iris melanomas and a single $(9 \%)$ ciliary body melanoma harbored KIT exon 11 mutations. No mutations were identified in conjunctival melanomas. The distribution of KIT and PDGFRA mutations by ocular melanoma anatomical site did not reach statistical significance $(P=0.393)$ CD117 positivity was not predictive of $K I T$ mutational status as only 6 of $58(10 \%)$ CD177-positive tumors harbored KIT mutations. In addition, a $K I T$ exon 17 mutation was identified in one CD117-negative tumor. KIT and PDGFRA mutations do occur in ocular melanomas at a frequency (11\%) that is similar to acral and mucosal melanomas. Limited correlation of CD117 positivity with mutational status suggests that all ocular melanomas should undergo mutational analysis to determine if imatinib therapy is appropriate.

Modern Pathology (2011) 24, 1031-1035; doi:10.1038/modpathol.2011.57; published online 8 April 2011
\end{abstract}

Keywords: CD117; KIT; ocular melanoma; PDGFRA

KIT signaling has been known to be important in melanocyte biology for approximately two decades. ${ }^{1-5}$ However, the role of KIT during melanoma pathogenesis has been contentious and is still not fully elucidated. Early studies of murine models and observations that KIT expression was lost in the dermal component of invasive melanomas led to the hypothesis that melanocytes leaving the epidermal boundaries would lose KIT expression., ${ }^{1,4,5}$ Thus, the invasive component of malignant melanomas would not demonstrate KIT expression but the

Correspondence: Dr LJ Layfield, MD, Department of Pathology, 1950 Circle of Hope, Rm 6755, Salt Lake City, UT 84112, USA. E-mail: layfiel@aruplab.com

Received 30 December 2010; revised 18 February 2011; accepted 18 February 2011; published online 8 April 2011 in situ/junctional component would express KIT. Our initial investigation into the role of KIT signaling in malignant melanomas confirmed that the majority of KIT expression was limited to in situ melanomas and the junctional component of invasive lesions. ${ }^{6}$ However, several metastatic melanomas in our study not only expressed KIT in their invasive components, but harbored KIT-activating mutations as well. Subsequently, others confirmed the presence of KIT mutations in the invasive portion of primary malignant melanomas and in their metastatic deposits. ${ }^{7-9}$ These findings suggested that targeted molecular therapy with tyrosine kinase inhibitors could be successful for melanoma, which is a notoriously difficult malignancy to treat. Indeed, promising results have emerged from clinical studies investigating the use of imatinib for 
melanoma patients with confirmed KIT-activating mutations. ${ }^{10-13}$

The prevalence of KIT mutations varies between melanoma subtypes, which are defined by the site of anatomical origin. Acral, mucosal, and chronic sun damaged skin melanomas have been shown to harbor KIT mutations while non-chronic sun damaged skin melanomas generally do not since they are often characterized by $B R A F$ or NRAS mutations. ${ }^{7-9,14,15}$ The frequency of KIT-activating mutations in specific melanoma subtypes is relatively low with reports of $15 \%$ for acral, $19 \%$ for mucosal, and $17 \%$ for chronic sun damaged skin melanomas. ${ }^{15,16}$ Ocular melanoma, although a rare melanoma subtype, does account for the majority of all intraocular malignancies. Arising from melanocytes of the choroid, iris, ciliary body, and conjunctiva, ocular melanomas have been reported to express KIT (CD117) at frequencies between 63 and 91\%. ${ }^{17-22}$ KIT mutations, however, have only been reported in one conjunctival tumor. ${ }^{8}$ Given the low frequency of KIT mutations $(<1 \%)$ in ocular melanoma, it is not surprisingly that recent studies have failed to demonstrate the clinical efficacy for imatinib mesylate therapy in unselected patients. ${ }^{6,18}$

As there are only limited data on the prevalence of KIT mutations in ocular melanoma, we reviewed a rather large series of 75 ocular melanomas including cases arising in the choroid, iris, ciliary body, and conjunctiva. The frequency of KIT-activating mutations and KIT protein expression was determined for each anatomical site. In addition, we also investigated the frequency of platelet-derived growth factor receptor A (PDGFRA) mutations in ocular melanoma, which has not been reported to date. Herein, we report the findings of that study.

\section{Materials and methods}

\section{Sources of Tissue}

Seventy-five ocular melanoma cases were retrieved from the files of the Department of Ophthalmology at the University of Utah. All cases were in-house cases and consecutively accessioned over a 15-year period. Cases were reviewed to confirm the original diagnosis and to ensure that sufficient tumor was present for analysis. The use of human tissue for this analysis was approved by the University of Utah Institutional Review Board.

\section{DNA Extraction}

Unstained slides from formalin-fixed paraffin-embedded tissue blocks were deparaffinized and rehydrated prior to manual microdissection of the tumor. Genomic DNA was isolated by an overnight proteinase $\mathrm{K}$ digestion. Following heat inactivation of the proteinase $K$, the crude extract was used directly in PCR.

\section{High-Resolution Melting Analysis and Sequencing}

These procedures have been described previously. ${ }^{23}$ Briefly, KIT exons 9, 11, 13, and 17 and PDGFRA exons 12 and 18 were screened for activating mutations by PCR and high-resolution melting analysis on a LightScanner32 (Idaho Technology). Each $10 \mu \mathrm{l}$ PCR reaction contained 5X master mix (0.2 U/ $\mu$ l KlenTaq1 DNA polymerase (AB Peptides), $44 \mathrm{ng} / \mu \mathrm{l}$ TaqStart Antibody (Clontech), $2.5 \mathrm{mg} / \mathrm{ml}$ bovine serum albumin (Sigma), $1 \mathrm{mM}$ each nucleotide mix (dATP, dCTP, dGTP, dTTP) (Roche), $10 \mathrm{mM}$ $\mathrm{MgCl}_{2}$ (Sigma), 5X LCGreen PLUS (Idaho Technology), and $250 \mathrm{mM}$ Tris $\mathrm{pH} 8.3$ ), $0.5 \mu \mathrm{M}$ forward primer, $0.5 \mu \mathrm{M}$ reverse primer, and $1 \mu \mathrm{l}$ crude DNA extract. Additional BSA $(1.2 \mu \mathrm{g} / \mu \mathrm{l})$ was added to heavily pigmented samples. PCR included an initial activation of $95^{\circ} \mathrm{C}$ for $120 \mathrm{~s}$, followed by 45 cycles of $95^{\circ} \mathrm{C}$ for $5 \mathrm{~s}, 62^{\circ} \mathrm{C}$ for $5 \mathrm{~s}$, and $74^{\circ} \mathrm{C}$ for $15 \mathrm{~s}$. Melting curves were generated by heating samples from 65 to $95^{\circ} \mathrm{C}$. Samples with abnormal melting curves, as determined by comparison to an internal negative control, were evaluated by Sanger sequencing at the University of Utah DNA sequencing core facility. The following Genbank reference sequences were utilized: KIT (HSU63834) and PDGFRA (M21574).

\section{CD117 Staining}

Immunohistochemical staining for KIT protein was performed with the rabbit polyclonal CD117 antibody from Dako Cytomation (Carpinteria, CA, USA). Automated staining was performed on $4-\mu \mathrm{m}$ thick sections of formalin-fixed, paraffin-embedded tissues. Sections were air-dried and then melted in a $60^{\circ} \mathrm{C}$ oven for $30 \mathrm{~min}$. Slides were loaded onto the Ventana (Tucson, AZ, USA) XT automated staining instrument where deparaffinization with EZ Prep solution and application of primary antibody (1:200; $32 \mathrm{~min}$ at $37^{\circ} \mathrm{C}$ ) were performed. The slides were detected using the IView Enhanced Alkaline Phosphatase RED detection kit (Ventana) and counterstained for 4 min with hematoxylin.

\section{Statistics}

The Fisher's exact test was used to correlate ocular melanoma anatomical location with CD117 positivity and KIT/PDGFRA mutational status.

\section{Results}

Fifty-eight of the $75(77 \%)$ ocular melanomas displayed at least weak focal staining with CD117. The distribution of CD117 positivity by anatomical site indicated that the prevalence of KIT expression was approximately equal in melanomas of the choroid, iris, and ciliary body $(81,83$, and $82 \%$, respectively) (Table 1). KIT expression was the least 
prevalent in conjunctival melanomas (20\%), but this failed to reach statistical significance $(P=0.641)$.

KIT and PDGFRA mutational analysis of the 75 ocular melanomas in our series identified 7 (9\%) with KIT mutations and 1 (1\%) with an intronic PDGFRA mutation. In total, 8 of 75 (11\%) ocular melanomas in our series had mutations in either KIT or PDGFRA. Five of 53 (9\%) choroidal, two of six $(33 \%)$ iris, and 1 of $11(9 \%)$ ciliary body melanomas had mutations (Table 1). No mutations were identified in our five conjunctival melanomas. The distribution of KIT and PDGFRA mutations by ocular melanoma anatomical site did not reach statistical significance $(P=0.393)$. Six of the eight mutations occurred in KIT exon 11 of which 2 were identical (P573L) while the remaining 4 were unique (Table 2). One of these cases harbored a nonsense mutation while two cases harbored two missense mutations, alleles unknown. One mutation each was present in KIT exon 17 (R804W) and PDGFRA intron 18.

Correlation of CD117 positivity with KIT mutational analysis revealed that 6 of 58 (10\%) CD117positive cases displayed a mutation in the KIT gene. All six of the mutations were located in KIT exon 11. One additional CD117-positive case harbored a mutation in PDGFRA intron 18. Of the 17 nonreactive CD117 cases, only one (6\%) harbored a KIT mutation, which was in exon 17 . None of the CD117-negative cases harbored PDGFRA mutations. Fifty-two of 75 (69\%) ocular melanomas demonstrated CD117 positivity but lacked mutations in KIT. These results indicate that CD117 positivity is a poor predictor of KIT mutational status.

\section{Discussion}

KIT mutations have been identified in $\sim 15 \%$ of acral, mucosal, and chronic sun damaged melanomas. ${ }^{6-9,14,15}$ Most of these reported mutations are predicted to be sensitive to tyrosine kinase inhibitors. Indeed, clinical studies using imatinib in melanoma patients with confirmed KIT-activating mutations have yielded promising results. ${ }^{10-13}$ Although it is arguably premature, the reported high frequency of KIT overexpression in ocular melanoma $(63-91 \%)^{17-22}$ has led to an interest in the use of imatinib therapy for this melanoma subtype. In initial studies, however, overexpression of KIT protein in ocular melanoma does not appear to imply response to imatinib. ${ }^{18,24}$ Currently, the cause of this insensitivity of ocular melanomas to imatinib is not entirely clear. The most plausible explanation is the near absence of KIT-activating mutations in ocular melanoma, as reported to date..$^{8,17,18,21}$ Only one conjunctival melanoma has demonstrated a KIT mutation. ${ }^{8}$ It is important to note that most studies have performed KIT mutational analysis on a small subset of CD117 expressing ocular tumors. ${ }^{17,18,21}$ The most extensive mutational analysis performed to date was by Beadling et $a l^{8}$ who examined 60 choroidal and 13 conjunctival melanomas. Our study set was of similar magnitude and included 53 choroidal, 5 conjunctival, 11 ciliary body, and 6 iris melanomas. We observed a KIT mutation frequency of $9 \%$ in our series of ocular melanomas, which is greater than the frequency of $1.4 \%$ demonstrated by Beadling et $a l^{8}$ Our study is the first to report KIT mutations

Table 1 KIT/PDGFRA mutations and CD117 expression in ocular melanoma by anatomical site

\begin{tabular}{|c|c|c|c|c|c|}
\hline Tumor site & $\begin{array}{c}\text { Percentage } \\
\text { with mutations }\end{array}$ & $\begin{array}{l}\text { Exons involved } \\
\text { (number of cases) }\end{array}$ & $\%$ CD117 positive & $\begin{array}{l}\% \text { Mutation+of } \\
\text { total CD117+ }\end{array}$ & $\begin{array}{l}\% \text { Mutation+of } \\
\text { total CD117- }\end{array}$ \\
\hline Choroidal & $9 \%(5 / 53)$ & $\begin{array}{c}\text { KIT exon } 11(3) \\
\text { KIT exon } 17(1) \\
\text { PDGFRA intron } 18(1)\end{array}$ & $81 \%(43 / 53)$ & $9 \%(4 / 43)$ & $10 \%(1 / 10)$ \\
\hline Iris & $33 \%(2 / 6)$ & KIT exon $11(2)$ & $83 \%(5 / 6)$ & $40 \%(2 / 5)$ & $0 \%(0 / 1)$ \\
\hline Ciliary body & $9 \%(1 / 11)$ & KIT exon 11 (1) & $82 \%(9 / 11)$ & $11 \%(1 / 9)$ & $0 \%(0 / 2)$ \\
\hline Conjunctiva & $0 \%(0 / 5)$ & N/A & $20 \%(1 / 5)$ & $0 \%(0 / 1)$ & $0 \%(0 / 4)$ \\
\hline
\end{tabular}

Table 2 KIT and PDGFRA mutations indentified in this study

\begin{tabular}{|c|c|c|c|c|}
\hline Mutation location & Ocular melanoma subtype & $\begin{array}{l}\text { Mutations deduced } \\
\text { at protein level }\end{array}$ & $\begin{array}{l}\text { Mutations identified } \\
\text { at DNA level }\end{array}$ & $\begin{array}{l}\text { Mutation type } \\
\text { at DNA level }\end{array}$ \\
\hline \multirow[t]{6}{*}{ KIT exon 11} & Choroidal & p.Val555Ile+p.Ser590Asn & c. $1684 \mathrm{G}>\mathrm{A}+\mathrm{c} .1790 \mathrm{G}>\mathrm{A}$ & Missense/missense \\
\hline & Choroidal & p.Val569Ile+p.Ser590Asn & c. $1726 \mathrm{G}>\mathrm{A}+\mathrm{c} .1790 \mathrm{G}>\mathrm{A}$ & Missense/missense \\
\hline & Iris & p.Asp572Asn & c. $1735 \mathrm{G}>\mathrm{A}$ & Missense \\
\hline & Iris & p.Pro573Leu & c. $1739 \mathrm{C}>\mathrm{T}$ & Missense \\
\hline & Choroidal & p.Pro573Leu & c. $1739 \mathrm{C}>\mathrm{T}$ & Missense \\
\hline & Ciliary body & p.Trp582X & c. $1767 \mathrm{G}>\mathrm{A}$ & Nonsense \\
\hline KIT exon 17 & Choroidal & p.Arg804Trp & c. $2431 \mathrm{C}>\mathrm{T}$ & Missense \\
\hline PDGFR intron 18 & Choroidal & & c. $2701+6 \mathrm{G}>\mathrm{A}$ & Splice site \\
\hline
\end{tabular}


in melanomas of the choroid, iris, and ciliary body. In combination with the data from Beadling et al, these data demonstrate that KIT mutations do occur in melanomas derived from all ocular anatomical locations.

The most commonly reported KIT mutation in acral, mucosal, and chronic sun damaged melanomas is L576P in exon $11 .^{6,7,9,15,25}$ While we did not observe the L576P mutation in any of our ocular melanomas, all but one of our KIT mutations were located in exon 11, which encodes the juxtamembrane domain of the receptor. It is widely believed that this domain normally functions as a negative regulator of kinase activity. Mutations that alter the conformation of this domain are therefore oncogenic due to the disruption of negative regulation. With the exception of W582X, we predict that the remaining exon 11 mutations (Table 2) are likely to be sensitive to imatinib. Conversely, the exon $17 \mathrm{R} 804 \mathrm{~W}$ mutation is likely to be imatinib resistant, given its location within the activation loop of KIT. However, additional in vitro studies would be needed to ultimately determine the efficacy of imatinib or other KIT inhibitors against these mutations.

In addition to KIT mutational analysis, we performed mutational analysis for the highly homologous receptor tyrosine kinase PDGFRA on our complete series of ocular melanomas. The PDGFRA gene, like KIT, is located at chromosome $4 \mathrm{q} 12$, which is a region that displays copy number variation in melanoma. ${ }^{9}$ Although no PDGFRA mutations have been reported to date in melanoma, ${ }^{7,9,26,27}$ no data are available for the ocular subtype. While we did not identify any PDGFRA mutations in exons 12 or 18 , we did identify an intron 18-point mutation in one CD117-positive choroidal melanoma. Given the location of the mutation near the exon/intron boundary, it is predicted to effect splicing. Additional studies would be needed to confirm that this mutation alters splicing and to evaluate whether it confers imatinib sensitivity. Our results suggest that PDGFRA mutations are extremely rare in ocular melanoma, which is in general agreement with the data from other melanoma subtypes. ${ }^{7,9,26,27}$

The utility of CD117 staining as a screen for KIT mutational status in ocular melanoma appears to be limited. Although 6 of 7 KIT mutated melanomas were CD117 positive in our study, the correlation failed to reach statistical significance. The majority of cases were CD117 positive and mutation negative (68\%), yielding a positive predictive value of only $12 \%$. One exon 17 mutation was discovered in a CD117-negative tumor, which would have been missed if mutational analysis was limited to only CD117-positive cases. Our results regarding the limited utility of CD117 staining in ocular melanoma are in accordance with the majority of data from other melanoma subtypes. Studies of acral, mucosal, and cutaneous melanomas by Beadling et al, ${ }^{8}$ Curtin et $a l^{9}$ and Rivera et $a l^{28}$ have all shown limited correlation between CD117 staining intensity and KIT mutational status. Although Torres-Cabala et $a l^{15}$ recently demonstrated a significant correlation between KIT mutational status and the percentage of CD117-positive tumor cells, irrespective of staining intensity, in their large series of acral, lentiginous, and mucosal melanomas, their positive predictive value was still low (14\%). Overall, this poor correlation between CD117 positivity and the presence of KIT-activating mutations may in part explain the non-responsiveness of these melanomas to imatinib therapy when patient eligibility is determined solely by CD117 status.

While we have demonstrated that KIT-activating mutations may be one method of driving KIT overexpression in ocular melanoma, additional mechanisms must also exist. Gene amplification, constitutive phosphorylation of the receptor, and dysfunction or deficiency of phosphatases that normally turn off the activated signal could all play a role. ${ }^{7,29}$ Alternatively, a decrease in protein turnover could result in the accumulation of KIT protein within neoplastic cells. In vitro studies have also implicated the stem cell factor/KIT autocrine loop in the malignant transformation of ocular melanocytes. ${ }^{17,19}$ Clearly, the role of KIT in the oncogenesis of ocular melanoma is not fully elucidated. However, the discovery of KIT-activating mutations in even a small proportion of ocular melanomas has important therapeutic implications for this malignancy, which is notoriously difficult to treat.

The present study is the first to show that KIT and PDGFRA mutations do occur in ocular melanomas at a frequency similar to that seen in acral, mucosal, and chronic sun damaged melanomas. Although no single mutation site appears to dominate, the majority of mutations are found in KIT exon 11. Melanomas of the iris may harbor KIT mutations more frequently than other anatomical sites but this correlation is hindered by a small sample size. CD117 immunoreactivity is not a good predictor for the presence of KIT mutations in ocular melanoma as only $10 \%$ of CD117-positive cases demonstrated a mutation. Screening of all ocular melanomas for KIT and PDGFRA mutations, irrespective of CD117 status, may be warranted to identify patients that could benefit from imatinib therapy.

\section{Disclosure/conflict of interest}

The authors declare no conflict of interest.

\section{References}

1 Alexeev V, Yoon K. Distinctive role of the ckit receptor tyrosine kinase signaling in mammalian melanocytes. J Invest Dermatol 2006;126:1102-1110. 
2 Grichnik JM. Kit and melanocyte migration. J Invest Dermatol 2006;126:945-947.

3 Grichnik JM, Burch JA, Burchette J, et al. The scf/kit pathway plays a critical role in the control of normal human melanocyte homeostasis. J Invest Dermatol 1998;111:233-238.

4 Montone KT, van Belle $\mathrm{P}$, Elenitsas $\mathrm{R}$, et al. Proto-oncogene c-kit expression in malignant melanoma: protein loss with tumor progression. Mod Pathol 1997;10:939-944.

5 Natali PG, Nicotra MR, Winkler AB, et al. Progression of human cutaneous melanoma is associated with loss of expression of c-kit proto-oncogene receptor. Int J Cancer 1992;52:197-201.

6 Willmore-Payne C, Holden JA, Tripp S, et al. Human malignant melanoma: detection of braf- and c-kitactivating mutations by high-resolution amplicon melting analysis. Hum Pathol 2005;36:486-493.

7 Antonescu CR, Busam KJ, Francone TD, et al. L576p kit mutation in anal melanomas correlates with kit protein expression and is sensitive to specific kinase inhibition. Int J Cancer 2007;121:257-264.

8 Beadling C, Jacobson-Dunlop E, Hodi FS, et al. Kit gene mutations and copy number in melanoma subtypes. Clin Cancer Res 2008;14:6821-6828.

9 Curtin JA, Busam K, Pinkel D, et al. Somatic activation of kit in distinct subtypes of melanoma. J Clin Oncol 2006;24:4340-4346.

10 Hodi FS, Friedlander P, Corless CL, et al. Major response to imatinib mesylate in kit-mutated melanoma. J Clin Oncol 2008;26:2046-2051.

$11 \mathrm{Kim} \mathrm{KB}$, Eton O, Davis DW, et al. Phase ii trial of imatinib mesylate in patients with metastatic melanoma. Br J Cancer 2008;99:734-740.

12 Lutzky J, Bauer J, Bastian BC. Dose-dependent, complete response to imatinib of a metastatic mucosal melanoma with a k642e kit mutation. Pigment Cell Melanoma Res 2008;21:492-493.

13 Quintas-Cardama A, Lazar AJ, Woodman SE, et al. Complete response of stage iv anal mucosal melanoma expressing kit val560asp to the multikinase inhibitor sorafenib. Nat Clin Pract Oncol 2008;5: 737-740.

14 Holden JA, Willmore-Payne C, Layfield LJ. Tyrosine kinase activating mutations in human malignancies: implications for diagnostic pathology. Exp Mol Pathol 2008;85:68-75.

15 Torres-Cabala CA, Wang WL, Trent J, et al. Correlation between kit expression and kit mutation in melanoma: a study of 173 cases with emphasis on the acral-lentiginous/mucosal type. Mod Pathol 2009;22:1446-1456.
16 Garrido MC, Bastian BC. Kit as a therapeutic target in melanoma. J Invest Dermatol 2010;130:20-27.

17 All-Ericsson C, Girnita L, Muller-Brunotte A, et al. C-kit-dependent growth of uveal melanoma cells: a potential therapeutic target? Invest Ophthalmol Vis Sci 2004;45:2075-2082.

18 Hofmann UB, Kauczok-Vetter CS, Houben R, et al. Overexpression of the kit/scf in uveal melanoma does not translate into clinical efficacy of imatinib mesylate. Clin Cancer Res 2009;15:324-329.

19 Lefevre G, Glotin AL, Calipel A, et al. Roles of stem cell factor/c-kit and effects of glivec/sti571 in human uveal melanoma cell tumorigenesis. J Biol Chem 2004;279:31769-31779.

20 Mouriaux F, Kherrouche Z, Maurage CA, et al. Expression of the c-kit receptor in choroidal melanomas. Melanoma Res 2003;13:161-166.

21 Pache M, Glatz K, Bosch D, et al. Sequence analysis and high-throughput immunohistochemical profiling of kit (cd 117) expression in uveal melanoma using tissue microarrays. Virchows Arch 2003;443:741-744.

22 Pereira PR, Odashiro AN, Marshall JC, et al. The role of c-kit and imatinib mesylate in uveal melanoma. J Carcinog 2005;4:19.

23 Wallander ML, Willmore-Payne C, Layfield LJ. C-kit and pdgfra zygosity in gastrointestinal stromal tumors: correlation with tumor site, tumor size, exon, and cd117 immunohistochemistry. Appl Immunohistochem Mol Morphol 2010;19:21-27.

24 Penel N, Delcambre C, Durando X, et al. O-mel-inib: a cancero-pole nord-ouest multicenter phase ii trial of high-dose imatinib mesylate in metastatic uveal melanoma. Invest New Drugs 2008;26:561-565.

25 Willmore-Payne C, Holden JA, Hirschowitz S, et al. Braf and c-kit gene copy number in mutation-positive malignant melanoma. Hum Pathol 2006;37:520-527.

26 Terada T. Low incidence of kit gene mutations and no pdgfra gene mutations in primary cutaneous melanoma: an immunohistochemical and molecular genetic study of Japanese cases. Int J Clin Oncol 2010;15: 453-456.

27 Curtin JA, Pinkel D, Bastian BC. Absence of pdgfra mutations in primary melanoma. J Invest Dermatol 2008;128:488-489.

28 Rivera RS, Nagatsuka H, Gunduz M, et al. C-kit protein expression correlated with activating mutations in kit gene in oral mucosal melanoma. Virchows Arch 2008;452:27-32.

29 Heinrich MC, Rubin BP, Longley BJ, et al. Biology and genetic aspects of gastrointestinal stromal tumors: kit activation and cytogenetic alterations. Hum Pathol 2002;33:484-495. 